\title{
An Energy Consumption Analytic Model for A Wireless Sensor MAC Protocol
}

\author{
Hung-Wei Tseng*, Shih-Hsien Yang*, Po-Yu Chuang*,Eric Hsiao-Kuang $\mathrm{Wu}^{\dagger}$, and Gen-Huey Chen* \\ * Dept. of Computer Science and Information Engineering, National Taiwan University, Taipei, Taiwan, R.O.C. \\ ${ }^{\dagger}$ Dept. of Computer Science and Information Engineering, National Central University, Chung-Li, Taiwan, R.O.C.
}

\begin{abstract}
A wireless sensor network is composed of a great number of sensing devices equipped with limited power sources. Under such battery-based constraint with limited power supply, energy consumption is a key issue for designing sensor network applications. Some sensor products adopt IEEE 802.11-like MAC protocol. However, IEEE 802.11 MAC is not a good solution for sensor networks. S(Sensor)-MAC proposes enhanced schemes such as periodic sleep and overhearing avoidance to provide a better choice for sensor applications. This paper presents an analytic model for evaluating the energy consumption at nodes in a S-MAC based wireless sensor network, and we develop an energy consumption analysis under different traffic conditions for distinct network topologies. The proposed model can guide designers to evaluate the energy consumption and determine the corresponding dominating factors. We validate the accuracy of the analytic model by comparing the analytic results with ns-2 simulation results.
\end{abstract}

\section{INTRODUCTION}

In recent years, wireless sensor network technology becomes an emerging field in wide range of applications such as environment monitoring, battlefield surveillance, telemonitoring of human physiological data, and etc. The low cost sensors with limited power sources can be deployed in differing places for differing usages, and they are able to sense temperature, fluid levels, humidity and other attributes. It is usually not cost effective to recharge the batteries of the sensors deployed on distinct environments; sensors are designed to carry limited irreplaceable power sources. Therefore, sensor network lifetime will rely on the the corresponding batteries of sensor nodes.

In many sensor network applications, sensor nodes are required to be low power to extend the lifetime of a network. Previous work [1] has shown that a large portion of power is consumed by the radio module, and the medium access control (MAC) layer usually coordinate the radio states. IEEE 802.11 is one of the most dominating MAC protocols in current times. However, the power control function of IEEE 802.11 can not satisfy the requirements of sensor network applications. Therefore, a great number of researchers proposed their modification on traditional IEEE 802.11 [2] [3] [4].

For the energy constraint of sensor applications, S(Sensor)MAC proposes several schemes improving traditional IEEE 802.11 in the aspect of energy consumption [2]. These schemes include periodic listening, overhearing avoidance, and message passing. In this paper, we propose an analytic model estimating energy consumption of sensor nodes adopting $\mathrm{S}$ MAC, and the accuracy of this model is also validated. We also present that the model can be applied to investigate the preferable design trade-off between energy and delay. On the other hand, the periodic listening scheme in S-MAC effectively avoids the idle listening problem in IEEE 802.11. By our model derivation, we find that such periodic listening scheme might cause unnecessary energy consumption in another aspect under heavy traffic load.

The rest of this paper is organized as follows. Section II discusses the related works.Section III provides the background of S-MAC. Section IV describes the proposed analytic model. Section $\mathrm{V}$ presents the analytic and simulation results to validate our model, and the application and some discussion of $\mathrm{S}-\mathrm{MAC}$ are also presented in the section. Section VI concludes this paper.

\section{RELATED WORK}

One of the most important design issues for sensor networks is energy management since sensor nodes are limited battery-based and cannot be recharged freely in most cases. Energy-aware medium access control schemes have received a great amount of attention from sensor network researchers since the MAC layer directly interfaces and collaborates with radio module, which consumes significant portion of device energies.

In the aspect of energy-aware sensor network, [3] proposed to save energy by using an extra ultra-low-power signaling channel that enables data channel to become into the sleep mode while neighboring nodes are in transmission, and such overhearing avoidance mechanism is also adopted in [2]. [4] also proposed the use of out-of-band signaling channel enabling data channel to sleep most of the time except data transmission, and the signaling channel also periodically sleeps to achieve better energy-saving.

Berkeley Motes are now widely adopted for sensor network application research field [5], but Motes use a simplified IEEE 802.11 protocol and support only one channel. As a result, out-of-band signaling protocols cannot be applied to Motes easily. S-MAC [2] studied in this paper proposed energy saving strategies with an in-band control method as traditional IEEE 802.11 does. To achieve better energy saving effects from idle listening of S-MAC, T-MAC [6] uses adaptive active scheme, which makes sensor nodes to transmit messages in bursts of variable length, and sleep between bursts.

Another problem arises in S-MAC is latency, which is also a critical consideration for particular sensor applications. To 
reduce the increased latency due to sleep delay in S-MAC, DMAC [7] proposed to deliver data along the data gathering tree, which significantly reduces the sleep latency. However, DMAC is only applicable under the specific data gathering tree scenario for unidirectional communication flow from multiple sources to a single sink.

In the aspect of IEEE 802.11-like protocol analysis, [8] presents detailed and accurate performance analysis of the IEEE 802.11 DCF scheme, which is widely used in ad hoc network, and a number of researchers construct their analytic models based on this paper for enhanced multi-service applications [9] [10] [11].

\section{S-MAC}

S-MAC [2] is a modification of IEEE 802.11 protocol specifically designed for sensor networks. In the conventional IEEE 802.11 protocol, there are several disadvantages causing unnecessary energy waste. First, in IEEE 802.11 standard, every node hears transmission of all neighboring nodes even if the packet is not destined to itself, which is known as overhearing. Besides, considerable control packets may increase overhead in energy consumption. The last one is idle listening, a node keeping listening to possible traffic for itself all the time. In typical sensor network applications, the traffic load is very low, that is, a node may not receive any traffic destined to it for a long period and the node is still in idle mode, which consumes power as much as in receiving mode [1].

To avoid the disadvantages of IEEE 802.11, S-MAC proposed corresponding energy-saving strategies. For the overhearing problem, S-MAC lets a node go to sleep for a period while receiving RTS or CTS packets not destined to itself, wherein the sleep period is the same as the duration in RTS or CTS packets. This scheme efficiently prevents a node from overhearing the following DATA and ACK packets. To reduce the number of control packets in IEEE 802.11, SMAC proposed a message passing mechanism which allows a number of fragments for a message to be transmitted with only one RTS and CTS. To reduce energy consumption in idle listening, S-MAC adopts a periodic listen and sleep schedule, which allows nodes turning off radio when in sleep mode. The nodes periodic synchronize schedules with their neighbors and creates virtual clusters where nodes in the same virtual cluster adopt the same listen and sleep schedule. A complete cycle of listen and sleep is called a frame in S-MAC. The portion of listen time in a frame is called duty cycle, which is introduced as a parameter in S-MAC.

\section{S-MAC ANALYTIC MODEL}

In this section, we give an analytical model of energy consumption for S-MAC described in [2].

Consider a time interval of period $t$, total energy consumption of a node running S-MAC during $t$ can be expressed as:

$$
E(t)=N_{T}(t) E_{T}+N_{R}(t) E_{R}+T_{S}(t) P_{S}+T_{I}(t) P_{I}
$$

where $N_{T}(t)$ and $N_{R}(t)$ denote numbers of times that a node transmits and receives a packet during $t$, respectively.
Moreover, $E_{T}$ and $E_{R}$ represent corresponding energy consumptions of transmitting and receiving a packet, respectively. Furthermore, $T_{S}(t)$ and $T_{I}(t)$ stand for the times the node spending in sleep mode and idle listening, respectively; therefore, $P_{S}$ is the power consumption of sleep mode, and $P_{I}$ is the power consumption of idle mode.

As referred in [2], when a node has a packet to transmit, carrier sense delay $\left(t_{C S}\right)$, backoff delay $\left(t_{B O}\right)$, transmission delay, propagation delay, processing delay, queueing delay, and sleep delay $\left(t_{S L}\right)$ will be considered. All the delays are the same as IEEE 802.11 protocol except sleep delay. Thus, these delays can be modelled as in IEEE 802.11 protocol, and several works have done on such models [8] [12], and we adopt these models for evaluating such delays. As described in [2], with the assumption that a packet arriving at the sender with equal probability within a frame, the sleep delay can be calculated as $\frac{T_{\text {frame }}}{2}$ where $T_{\text {frame }}$ stands for the period of a frame, the complete cycle of listen and sleep. During the sleep delay period, the node will stay in listening mode. Besides, the node will send RTS and data to destination, and receive CTS and ACK frames from destination node. Therefore, the energy consumption for transmitting a packet can be evaluated as :

$$
\begin{array}{r}
E_{T}=P_{T x}\left(t_{R T S}+t_{\text {data }}\right)+P_{R x}\left(t_{C S}+t_{B O}+t_{S L}\right. \\
\left.+t_{C T S}+t_{A C K}+3 t_{S I F S}+t_{D I F S}\right)
\end{array}
$$

where $P_{T x}$ and $P_{R x}$ are the power consumptions for a node in transmitting and receiving mode respectively, and $t_{R T S}$, $t_{\text {data }}, t_{C S}, t_{B O}, t_{S L}, t_{C T S}, t_{A C K}, t_{S I F S}$, and $t_{D I F S}$ are the times spent in sending RTS, sending data, carrier sense delay, backoff delay, sleep delay, receiving CTS, receivng ACK, SIFS, and DIFS, respectively. Similarly, the energy consumption for receiving a packet can be evaluated as:

$$
\begin{aligned}
E_{R}=P_{T x}\left(t_{C T S}+t_{A C K}\right)+ & P_{R x}\left(t_{R T S}+t_{\text {data }}\right. \\
& \left.+3 t_{S I F S}+t_{D I F S}\right)
\end{aligned}
$$

Assume that a node with Poisson arrival rate of transmitting packets $\lambda_{T}$, and Poisson arrival rate of receiving packets $\lambda_{R}$, then the number of times the node sends and receives packets during $t$ can be expressed as:

$$
\begin{aligned}
& N_{T}(t)=\lambda_{T} t \\
& N_{R}(t)=\lambda_{R} t
\end{aligned}
$$

We now consider the node with $n$ neighboring nodes within its radio range, and neighboring nodes are numbered with $1,2, \ldots, n$. The packet transmitting rates of neighbor $i$ is $\lambda_{T_{i}}$, packet receiving rates of neighbor $i$ is $\lambda_{R_{i}}$, and packet receiving rate of node $j$ from another neighbor $i$ is $\lambda_{R_{i j}}$. As describe in section III, a S-MAC sensor node goes into sleep mode in three cases. The first case is scheduled sleep time, the second case is receiving a RTS frame from its neighboring nodes, and the third case is receiving a CTS frame from its neighboring. In the last two cases, the node will sleep for a data transmission period recorded in RTS or CTS frames. Assume that a node receives RTS or CTS frames from its neighbors with probability $p_{\text {succ }}$. $T_{\text {listen }}$ and $T_{\text {sleep }}$ are the listen and 
sleep period during a complete listen-sleep cycle. The sleep time $T_{S}$ and the idle listening time $T_{I}$ during period $t$ can be expressed as:

$$
\begin{aligned}
T_{S}= & p_{\text {succ }}\left[\left(\left(\sum_{i=1}^{n} \lambda_{T_{i}}-\lambda_{R}\right) t\right) t_{\text {data }}+\right. \\
& \left.\left(\left(\sum_{i=1}^{n} \lambda_{R_{i}}-\sum_{i=1, j=1, i \neq j}^{n} \lambda_{R_{i j}}-\lambda_{T}\right) t\right) t_{\text {data }}\right] \\
& +\left[t-\left(\left(\left(\sum_{i=1}^{n} \lambda_{T_{i}}-\lambda_{R}\right) t\right) t_{\text {data }}+\right.\right. \\
& \left(\left(\sum_{i=1}^{n} \lambda_{R_{i}}-\sum_{i=1, j=1, i \neq j}^{n} \lambda_{R_{i j}}-\lambda_{T}\right) t\right) t_{\text {data }} \\
& +N_{T}\left(t_{R T S}+t_{\text {data }}+t_{C S}+t_{\text {BO }}+t_{S L}\right. \\
& \left.+t_{C T S}+t_{A C K}+3 t_{S I F S}+t_{D I F S}\right) \\
& +N_{R}\left(t_{R T S}+t_{\text {data }}+t_{C T S}+t_{\text {ACK }}\right. \\
& \left.\left.\left.+3 t_{S I F S}+t_{D I F S}\right)\right)-\epsilon\right] \times\left(\frac{T_{\text {sleep }}}{T_{\text {frame }}}\right) \\
T_{I}= & {\left[t-p_{\text {succ }}\left(\left(\left(\left(\sum_{i=1}^{n} \lambda_{T_{i}}-\lambda_{R}\right) t\right) t_{\text {data }}+\right.\right.\right.} \\
& \left.\left(\left(\sum_{i=1}^{n} \lambda_{R_{i}}-\sum_{i=1, j=1, i \neq j}^{n} \lambda_{R_{i j}}-\lambda_{T}\right) t\right) t_{\text {data }}\right) \\
& +N_{T}\left(t_{R T S}+t_{\text {data }}+t_{C S}+t_{\text {BO }}+t_{S L}\right. \\
& \left.+t_{C T S}+t_{A C K}+3 t_{S I F S}+t_{\text {DIFS }}\right) \\
& +N_{R}\left(t_{R T S}+t_{\text {data }}+t_{C T S}+t_{\text {ACK }}\right. \\
& \left.\left.\left.+3 t_{S I F S}+t_{D I F S}\right)\right)-\epsilon\right] \times\left(\frac{T_{\text {listen }}}{T_{\text {frame }}}\right)+\epsilon
\end{aligned}
$$

The $p_{\text {succ }}$ is related to the probability that a transmission is successful and the probability that the node is listening or not. The probability of successful transmission can be obtained as in [8]. In S-MAC, the sleep schedule for a node will be synchronized with all its neighbors, so we can assume that RTS/CTS control packets will be sent by the neighbors of the node only when the node wakes up. The assumption is quite reasonable just as described in [2]. By applying the probability of successful transmission, and assuming that each of the $n$ stations contending on the same channel with transmission probability $\tau$, we can derive $p_{\text {succ }}$ by the following formula:

$$
p_{\text {succ }}=\frac{n \tau(1-\tau)^{n-1}}{1-(1-\tau)^{n}}
$$

The symbol $\epsilon$ represents the total extra idle period when a node change its mode back to periodic listen and sleep schedule. Such extra idle period occurs when a node finishes a packet transmitting or receiving. Because the finishing time of transmitting or receiving packet is not always fit to scheduled listen and sleep time, the node must keep idle for such extra idle period until the scheduled listen or sleep time arrives. If the node plays a role as a router in the network, the node just forwards the packets immediately, and the extra idle occurs only when the node finishes forwarding packets. Similar to

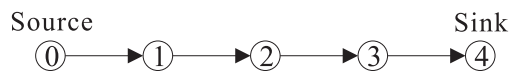

Fig. 1. The chain-hop topology used in validating our analytic model

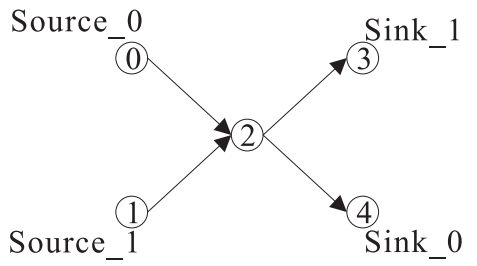

Fig. 2. Another topology used in validating our analytic model

sleep delay, since the packet transmission may also finish with equal probability within a frame, the extra idle period can be calculated as $\frac{T_{\text {frame }}}{2}$. Let $N_{I}$ be the number of times that the extra idle period occurs, the total extra idle time is:

$$
\epsilon=N_{I} \times \frac{T_{\text {frame }}}{2}
$$

By deriving the unknowns in (1) using (2)(3)(4)(5)(6)(7)(8) (9), we can evaluate the energy consumption of a node in analytic method.

\section{AnAlytic Result}

In this section, we will compare the simulation and analytic results to show the accuracy of our proposed model. We also shows how the model can be used to help sensor application design. Finally, we present some observations of S-MAC based on the proposed model.

\section{A. Model Validation}

We validate the analytic model of S-MAC by comparing the analytic results with simulation results. The simulation was done by using ns-2 network simulator. The MAC layer protocol used is S-MAC with overhearing avoidance and periodic sleep scheme. We also assume that all nodes adopt the same sleep schedule when making our analytic model, and

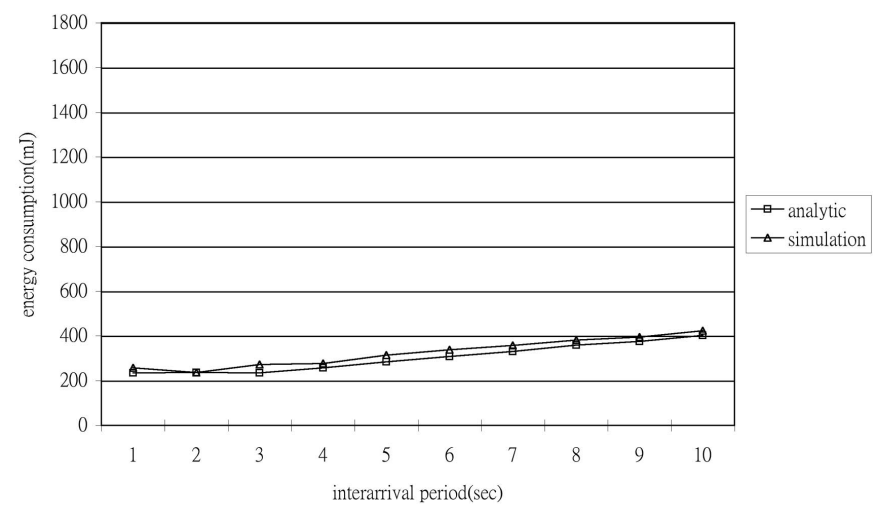

Fig. 3. The energy consumption of node 0 in Fig. 1 evaluated by analytic model and simulation 


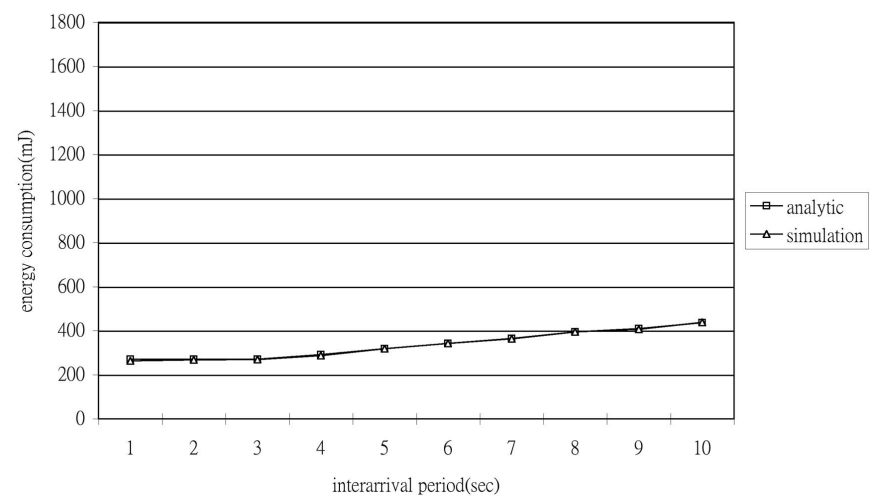

Fig. 4. The energy consumption of node 2 in Fig. 1 evaluated by analytic model and simulation

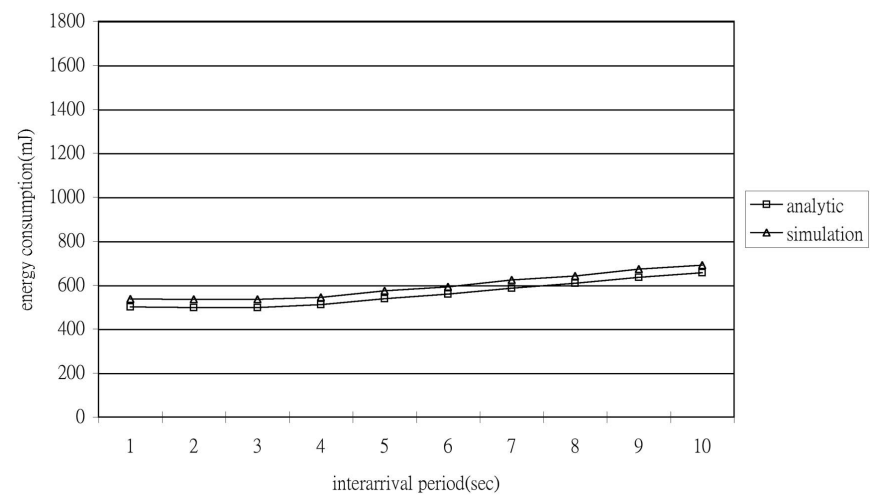

Fig. 5. The energy consumption of node 2 in Fig. 2 evaluated by analytic model and simulation

the $P_{T x}$ is $24.75 \mathrm{~mW}, P_{R x}$ and $P_{I}$ are $13.5 \mathrm{~mW}$, and $P_{S}$ is 15 $\mathrm{uW}$, just as the values used in [2]. The bandwidth is set to be $40 \mathrm{kbps}$. The messages are generated from the source node to the destination node, and each message is 400 Bytes in size. The parameters described above are also used in the following experiments presented in this section.

We set up two experiments to validate our model using topologies shown in Fig. 1 and Fig. 2. The message interarrival period is varying from $1 \mathrm{~s}$ to $10 \mathrm{~s}$, and the duration is the time that 10 messages have been transmitted to the sink. The duty cycle $\left(\frac{T_{\text {listen }}}{T_{\text {frame }}}\right)$ is set to $20 \%$. In the topology of Fig. 1, each node $i$ can receive radio only from its immediate neighbor $(i-1$ and $i+1)$. In the topology of Fig. 2, node 0, 1,3 , and 4 can only receive radio only from node 2 .

In the topology of Fig. 1, node 0 and node 2 are selected to compare our analytic and simulation results. Node 0 is the source node, which generates the traffic, and node 0 can hear RTS when node 1 transmitting to node 2 . Node 2 is an intermediate node which can hear RTS when node 3 transmitting to node 4 and CTS when node 0 transmitting to node 1. As shown in Fig. 3 and Fig. 4, the results generated by proposed model are quite approaching to the simulation results.

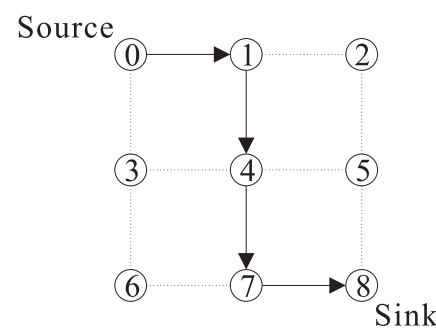

Fig. 6. A grid topology used in analysis S-MAC based sensor network design

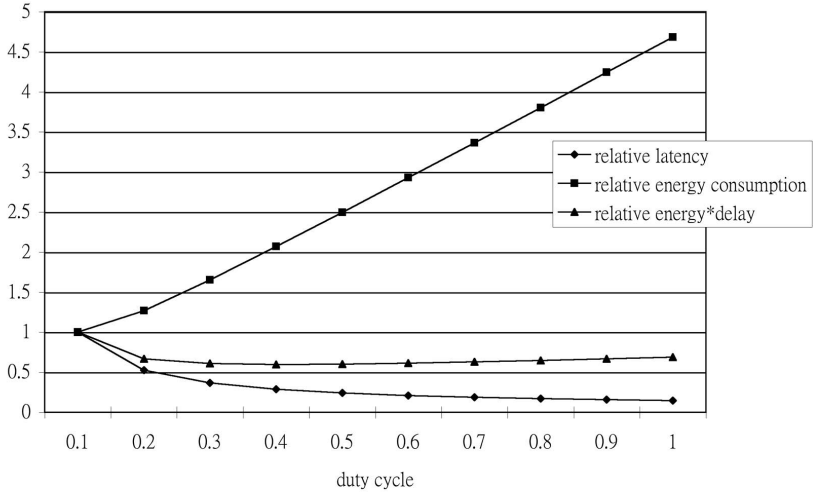

Fig. 7. Relative energy consumption, relative latency, and relative energy*delay of total network with duty cycle varying from 0.1 to 1.0 .1 duty cycle is the base

In the topology of Fig. 2, node 2 plays as a router, which receives packets from source node 0 (source_0) and node 1 (source_1) and forwards these packets to node 3 (sink_1) and node 4 (sink_0). Unlike any other nodes in Fig. 1 and Fig. 2, node 2 involves in every transmissions, that is, node 2 sleeps only during scheduled sleep time. The analytic and simulation results of node 2 in Fig. 2 are shown in Fig. 5, and results generated by proposed model are also quite approaching to the simulation results.

\section{B. Energy Efficiency of S-MAC}

In many sensor network applications, energy consumption is not the only important design issue. For example, in the environment surveillance system, the latency for an event transmitted from source to data sink would be very important, so that the system can take appropriate actions as soon as possible. Sometimes, energy and latency would be a tradeoff. To find out the balance between energy and latency, some researchers adopt energy*delay product as the metric to evaluate the design trade-off [7] [13].

Periodic sleep scheme in S-MAC saves energy while nodes are idle, but this scheme also introduces additional delay for waiting the receiver back to listening from sleeping. Duty cycle is the parameter standing for the portion of time a node is listening in a frame. Therefore, duty cycle is the factor that influences the effect of energy saving in idle listening. However, short duty cycle would result in longer sleep delay, 


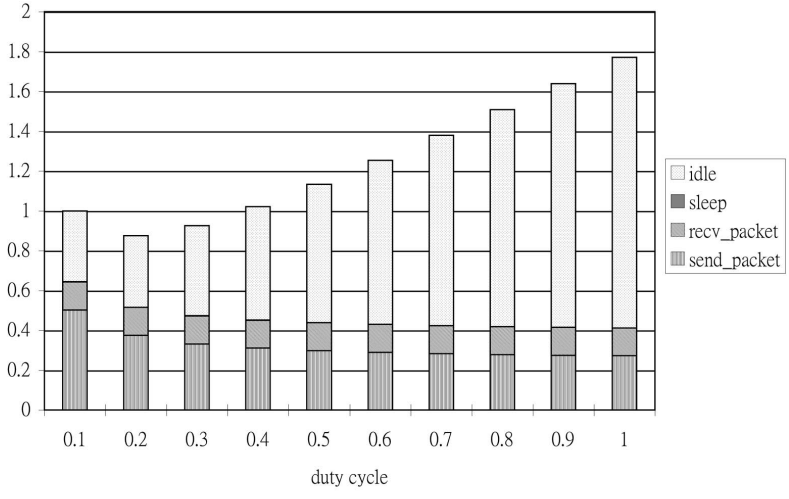

Fig. 8. Relative energy consumption of total network of 20 chain-hops. The duty cycle varies from 0.1 to 1.0 .1 duty cycle is the base

because the packets may arrive when receiver is sleeping with a higher probability. That is, choosing a shorter duty cycle would decrease the idle listening time, but increase the latency. With our analytic model, we can estimate the energy consumption under different duty cycles for sensor nodes and evaluate the design trade-offs easily.

Fig. 6 shows a portion of an exemplary sensor network topology where node 0 is the data source, and the data are transmitted along the path of node 1 , node 4 , and node 7 to the destination data sink node 8. The nodes in Fig. 6 can transmit or receive packets to or from the neighbor nodes which connected by hidden lines or arrows in the figure. The packets are generated from data source every 10 seconds, and each message is sized in 400 Bytes.

Fig. 7 shows the relative energy consumption, relative latency, and relative energy*delay of total network with duty cycle varying from 0.1 to 1 , and 0.1 duty cycle is chosen as the base. As shown in Fig. 7, we can see that the longer the duty cycle is, the more energy consumes since shorter duty cycle saves more energy from idle listening. In contrast, the latency becomes shorter when duty cycle is longer. The best energy*delay balance appears at the point where duty cycle is 0.4 .

According to formula (1), the energy consumption of $\mathrm{S}$ MAC based sensor nodes can be divided into four parts: sending packets, receiving packets, idle listening, and sleeping. In order to explore the performance of S-MAC under heavy traffic, we also set up and analyze another scenario. The scenario forms like Fig. 1, but the number of hops is increased to 20 hops. Fig. 8 shows the relative total energy consumption of the 20-hop-chain sensor network, and energy consumption of each duty cycle is divided into four parts corresponding to the energy consumption in sending packets, receiving packets, idle listening, and sleeping. Each message is sized in 400 Bytes, and the interarrival period of messages is set to 5 seconds, which the traffic is heavier than 10 seconds in the above cases. The lowest energy consumption holds when duty cycle is 0.2 , not 0.1 , which saves the most idle listening energy. Due to much longer sleep delay, the nodes must spend more time in sending packets to wait for the receiver back to listening mode, and this wastes energy of sender nodes. The results also points out that while the network traffic is heavy, shorter duty cycle may not always result in better energy saving.

\section{CONCLUSIONS AND FUTURE WORK}

S-MAC have received considerable attention due to its energy saving schemes designed for sensor network. This paper introduces an analytical model to estimate the energy consumption of S-MAC. Our model shows its accuracy by comparing with NS-2 simulation results. With the help of our model, sensor network application designers can evaluate the energy efficiency of their S-MAC based sensor network under different parameters, such as duty cycles, interarrival periods, and etc.

S-MAC saves energy, but sacrifices latency. To achieve better energy efficiency than S-MAC, the future work includes the improvement of energy consumption and the reduction of packet latency of S-MAC.

\section{ACKNOWLEDGMENT}

This work was supported by the MediaTek Inc. under the project "Wireless Communication Systems".

\section{REFERENCES}

[1] M. Stemm and R. H. Katz, "Measuring and reducing energy consumption of network interfaces in hand-held devices," IEICE Transactions on Communications, vol. E80-B, no. 8, pp. 1125-31, 1997.

[2] W. Ye, J. Heidemann, and D. Estrin, "An energy-efficient MAC protocol for wireless sensor networks," in Proceedings of IEEE INFOCOM, June 2002, pp. 1567-1576.

[3] S. Singh and C. Raghavendra, "PAMAS: Power aware multi-access protocol with signalling for ad hoc networks," ACM Computer Communication Review, vol. 28, no. 3, pp. 5-26, July 1998.

[4] C. Schurgers, V. Tsiatsis, S. Ganeriwal, and M. Srivastava, "Optimizing sensor networks in the energy-latency-density design space," IEEE Transactions on Mobile Computing, vol. 1, pp. 70-80, January 2002.

[5] "University of california, berkeley, mica2 schematics," March 2003. [Online]. Available: http://webs.cs.berkeley.edu/tos/hardware/ design/ORCAD_FILES/MICA2/6310-0306-01ACLEAN.pdf

[6] "An adaptive energy efficient MAC protocol for wireless sensor networks," in Proceedings of the First ACM Conference on Embedded Networked Sensor Systems, November 2003, pp. 171-180.

[7] "An adaptive energy-efficient and low-latency MAC for data gathering in sensor networks," in Proceedings of 18th International Parallel and Distributed Processing Symposium, April 2004, pp. 224-231.

[8] G. Bianchi, "Performance analysis of the IEEE 802.11 distributed coordination function," IEEE Journal of Selected Areas in Communication, vol. 18, no. 3, pp. 535-547, March 2000.

[9] L. Chandran-Wadia, S. Mahajan, and S. Iyer, "Redefining internet in the context of pervasive computing," in Proceedings of the 15th international conference on Computer communication, 2002, pp. $36-$ 49.

[10] Y. Xiao and J. Rosdahl, "Performance analysis and enhancement for the current and future IEEE 802.11 MAC protocols," ACM SIGMOBILE Mobile Computing and Communications Review, vol. 7, pp. 6-19, 2003.

[11] H. Wu, Y. Peng, K. Long, S. Cheng, and J. Ma, "Performance of reliable transport protocol over IEEE 802.11 wireless LAN: Analysis and enhancement," in Proceedings of IEEE INFOCOM, vol. 21, no. 1, June 2002, pp. 599 - 607.

[12] "Queueing analysis and delay mitigation in IEEE 802.11 random access MAC based wireless networks," in proceedings of IEEE INFOCOM, March 2004.

[13] "Data gathering in sensor networks using the energy*delay metric," in Proceedings of the IPDPS Workshop on Issues in Wireless Networks and Mobile Computing, April 2001. 